\title{
The Evolving Clinical Presentation of Acute Rejection in Facial Transplantation
}

Valentin Haug, MD; Branislav Kollar, MD; Doha Obed, CandMed; Harriet Kiwanuka, BA; Marvee Turk, MD; Luccie Wo, MD; Sotirios Tasigiorgos, MD; Maximillian Kueckelhaus, MD; Leonardo V. Riella, MD, PhD; Bohdan Pomahac, MD

IMPORTANCE Acute rejection is one of the most frequent complications in facial transplantation, with potentially severe consequences for the recipient if overlooked. Clinical signs, such as erythema or edema, are helpful to diagnose acute rejection in the early follow-up stage; however, it is not well known whether these clinical signs remain reliable markers of acute rejection beyond the second posttransplant year.

OBJECTIVE To determine the diagnostic value of clinical signs of acute rejection after facial transplantation over time.

DESIGN, SETTING, AND PARTICIPANTS A retrospective, single-center cohort study was conducted of patients who underwent facial transplantation at Brigham and Women's Hospital between April 2009 and October 2014, with up to an 8-year follow-up. Medical records were reviewed until September 30, 2017. The medical records from 104 encounters with 7 patients who underwent partial or full facial transplantation were analyzed for symptoms of rejection, immunosuppressive therapy, and histopathologic findings.

MAIN OUTCOMES AND MEASURES The occurrence of 5 clinical signs of acute rejection were evaluated: erythema, edema, exanthema, suture line erythema, and mucosal lesions. Odds ratios (ORs) were calculated to determine the statistically significant association of these signs with the histopathologic diagnosis of rejection. In addition, tacrolimus blood levels, as a surrogate marker of immunosuppressive therapy, were evaluated.

RESULTS Of the 7 patients included in the study, 5 were men. The mean follow-up was 66 months (range, 35-101). Of 104 clinical encounters, 46 encounters (44.2\%) represented rejection episodes and 58 encounters $(55.8 \%)$ represented no-rejection episodes. Beyond 2 years posttransplantation, only erythema $(\mathrm{OR}, 6.53 ; 95 \% \mathrm{Cl}, 1.84-20.11 ; P=.004)$ and exanthema $(\mathrm{OR}, \infty ; 95 \% \mathrm{Cl}, 2.2-\infty ; P=.004)$ were demonstrated to be reliable clinical signs of acute rejection in facial transplantation. There was also a statistically significant association of subtherapeutic tacrolimus levels with late rejection episodes (OR, 3.79; 95\% Cl, 1.25-12.88; $P=.03$ ). In addition, the occurrence of subclinical rejection was more frequent during later follow-up times (7 [24.1\%] late rejections vs 1 [5.9\%] early rejection). Five of 8 subclinical rejections (62.5\%) were associated with subtherapeutic tacrolimus levels.

CONCLUSIONS AND RELEVANCE Clinical signs of acute rejection in facial transplantation appear to be of limited diagnostic value, particularly after the second postoperative year. Until alternative biomarkers for rejection are identified, protocol skin biopsies will remain necessary for guiding assessments of allograft rejection.

LEVEL OF EVIDENCE 3.

Author Affiliations: Division of Plastic Surgery, Department of Surgery, Brigham and Women's Hospital, Harvard Medical School, Boston, Massachusetts (Haug, Kollar, Obed, Kiwanuka, Turk, Wo, Tasigiorgos, Kueckelhaus, Pomahac) Department of Hand, Plastic and Reconstructive Surgery, Burn Trauma Center, BG Trauma Center Ludwigshafen, University of Heidelberg, Ludwigshafen, Germany (Haug); Schuster Transplantation Research Center, Brigham and Women's Hospital, Harvard Medical School, Boston, Massachusetts (Riella).

Corresponding Author: Bohdan Pomahac, MD, Division of Plastic Surgery, Department of Surgery. Brigham and Women's Hospital, Harvard Medical School 75 Francis St, Boston, MA 02115 (bpomahac@bwh.harvard.edu). 
$\mathrm{F}$ acial transplantation (FT) is a promising treatment option for patients with devastating injuries to the face. ${ }^{1,2}$ To date, more than 40 FTs have been performed worldwide with encouraging outcomes. ${ }^{3}$ In skin-bearing, vascularized composite allotransplantations, such as FT, one of the most frequent complications is acute rejection (AR), occurring in more than $80 \%$ of patients within the first postoperative year. ${ }^{4}$

The current standard to diagnose AR is the evaluation of biopsy findings according to Banff classification along with clinical presentation of the allograft. ${ }^{5}$ Acute rejection in FT has been described to entail a broad spectrum of clinical findings, such as diffuse erythema, soft tissue edema, maculopapular rash, or mucosal involvement. ${ }^{6-9}$ Because the face can be directly inspected, the obvious clinical signs of rejection might be partially responsible for the high rates of diagnosis as well as early treatment of AR in FT. ${ }^{10}$ Therefore, the clinical signs are considered to be particularly helpful in diagnosing AR in FT in the first 2 postoperative years.

However, the diverse macroscopic manifestation reflects the complexity of the dermal response to inflammation, and these intraindividual and interindividual differences in AR can make a clinical diagnosis challenging. Many inflammatory or infectious conditions of the skin may be clinically and histologically indistinguishable from AR in FT. ${ }^{11}$ Furthermore, the Banff system is derived from skin samples from upper extremity allografts and does not overtly account for the distinct immunologic milieu that characterizes skin from the face. ${ }^{12}$

Because quantitative outcomes of FT beyond 2 years posttransplantation are scarce, it remains to be elucidated whether the clinical signs of AR remain reliable markers of rejection over time. In an effort to appraise the diagnostic value of the diversely reported clinical signs of AR, we performed a singlecenter, retrospective cohort study of our experience with immune monitoring in $7 \mathrm{FT}$ recipients with up to 8 years of followup. More specifically, we wanted to investigate how the clinical signs of AR in FT evolve compared with skin biopsy findings according to the Banff classification.

\section{Methods}

\section{Study Design}

We conducted a retrospective study of 7 patients who underwent full or partial FT at Brigham and Women's Hospital, Boston, Massachusetts, between April 2009 and October 2014. Medical records were reviewed until September 30, 2017. All patients provided written informed consent to participate in the clinical study for FT, as approved by the Brigham and Women's Hospital Institutional Review Board and by the Army Medical Research and Material Command Human Research Protection Office. All study participants also provided written informed consent for publication of their identifiable clinical photographs.

\section{Immunosuppression}

The details about immunosuppressive protocols were published previously. ${ }^{13,14}$ Briefly, therapy was induced with antithymoglobulin, $1.5 \mathrm{mg} / \mathrm{kg} / \mathrm{d}$ for 4 days and single doses of

\section{Key Points}

Question Do clinical signs, such as erythema or edema, remain reliable markers of acute rejection in facial transplantation over time?

Findings This single-center analysis of 7 patients who underwent facial transplantation with up to 8 years of follow-up demonstrated that only erythema and exanthema are reliable clinical signs with significant association with acute rejection beyond the second posttransplant year. Subclinical rejections occurred more frequently during later follow-up times.

Meaning Acute rejection in facial transplantation becomes less macroscopically ascertainable and accordingly more difficult to diagnose over time; thus, protocol biopsies for immune monitoring remain necessary to complement the allograft rejection diagnosis.

mycophenolate mofetil, $1 \mathrm{~g}$, and methylprednisolone, $500 \mathrm{mg}$, were given before surgery. The maintenance immunosuppression then typically consisted of triple therapy with tacrolimus, mycophenolate mofetil, and corticosteroids. Furthermore, we attempted to withdraw corticosteroid therapy and reduce mycophenolate mofetil doses. ${ }^{15}$ The target tacrolimus trough levels were as follows: 0 to 6 months, 10 to $15 \mathrm{ng} / \mathrm{mL}$; 7 to 12 months, 8 to $12 \mathrm{ng} / \mathrm{mL}$; and longer than 12 months, 6 to $10 \mathrm{ng} / \mathrm{mL}$. From postoperative month 14 , patient 6 received belatacept, $5 \mathrm{mg} / \mathrm{kg} / \mathrm{mo}$, and his tacrolimus target trough levels were reduced to 4 to $5 \mathrm{ng} / \mathrm{mL} .{ }^{16}$ Accordingly, tacrolimus trough levels below the target levels, as outlined, were defined as subtherapeutic. Acute T-cell-mediated rejection (TCMR) was managed with 1 or a combination of treatment strategies: adjustment of maintenance immunosuppression, topical therapy, or pulsed systemic corticosteroids. In case of corticosteroidrefractory TCMR, antithymoglobulin and alemtuzumab use was further considered. For the treatment of antibodymediated rejection (AMR), more extensive therapeutic modalities (eg, plasmapheresis, bortezomib, or eculizumab) were applied. ${ }^{17}$

\section{Diagnosis of Rejection}

Face allograft biopsies (4-mm skin punch) were performed at 3,6 , and 12 months and then yearly as well as during suspected rejection episodes. The formalin-fixed, paraffinembedded biopsies were sectioned and routinely stained with hematoxylin and eosin stain. Acute TCMR was diagnosed in accordance with the Banff classification of skin-containing composite tissues with the following grades: 0 , no or rare inflammatory infiltrates; I (mild), mild perivascular infiltration (with no epidermal involvement); II (moderate), moderate to severe perivascular inflammation with or without mild epidermal and/or adnexal involvement; III (severe), dense inflammation and epidermal involvement with epithelial apoptosis, dyskeratosis, and/or keratinolysis; and IV (necrotizing AR), frank necrosis of epidermis or other skin structures. ${ }^{5}$ The biopsy grade was determined as a consensus opinion of at least 2 experienced, independent dermatopathologists. The biopsy results were further discussed at intradisciplinary and 


\begin{tabular}{|c|c|c|c|c|c|c|c|}
\hline \multirow[b]{2}{*}{ Characteristic } & \multicolumn{7}{|l|}{$\underline{\text { Patient }}$} \\
\hline & 1 & 2 & 3 & 4 & 5 & 6 & 7 \\
\hline $\begin{array}{l}\text { Year of } \\
\text { transplantation }\end{array}$ & 2009 & 2011 & 2011 & 2011 & 2013 & 2014 & 2014 \\
\hline $\begin{array}{l}\text { Age at } \\
\text { transplantation, y }\end{array}$ & Late $50 \mathrm{~s}$ & Mid-20s & Early 30 s & Late 50 s & Mid-40s & Late 30s & Early 30 s \\
\hline Sex & Male & Male & Male & Female & Female & Male & Male \\
\hline Race & White & White & White & White & White & White & White \\
\hline $\begin{array}{l}\text { Mechanism of } \\
\text { injury }\end{array}$ & Electrical burn & Electrical burn & Electrical burn & Animal attack & Chemical burn & Ballistic trauma & Ballistic trauma \\
\hline Facial defect & $\begin{array}{l}\text { Missing nose, } \\
\text { maxilla, and } \\
\text { upper lip }\end{array}$ & $\begin{array}{l}\text { Complete } \\
\text { absence of all } \\
\text { facial } \\
\text { structures }\end{array}$ & $\begin{array}{l}\text { Diffuse scarring, } \\
\text { missing nose, } \\
\text { parts of upper } \\
\text { and lower lips }\end{array}$ & $\begin{array}{l}\text { Missing bilateral } \\
\text { eyelids and eyes, } \\
\text { nose, maxilla, } \\
\text { upper and } \\
\text { lower lips }\end{array}$ & $\begin{array}{l}\text { Diffuse scarring, } \\
\text { missing bilateral } \\
\text { eyelids, upper } \\
\text { and lower lips }\end{array}$ & $\begin{array}{l}\text { Missing nose, } \\
\text { maxilla, } \\
\text { mandible, } \\
\text { upper and } \\
\text { lower lips }\end{array}$ & $\begin{array}{l}\text { Missing nose, } \\
\text { maxilla, } \\
\text { mandible, } \\
\text { upper and } \\
\text { lower lips }\end{array}$ \\
\hline Graft type & Partial face & Full face & Full face & Full face & Full face & Partial face & Partial face \\
\hline Ischemia time, h & 2 & 4 & 2 & 2 & 3 & 3 & 1.5 \\
\hline PRA, \% & NA & 68 & 0 & 0 & 97 & 22 & 32 \\
\hline DSA & Negative & Negative & Negative & Negative & Positive & Negative & Positive \\
\hline $\begin{array}{l}\text { HLA mismatch } \\
(A, B, D R)\end{array}$ & $3 / 6$ & $4 / 6$ & $4 / 6$ & $5 / 6$ & $5 / 6$ & $5 / 6$ & $5 / 6$ \\
\hline $\begin{array}{l}\text { CMV } \\
\text { (donor/recipient) }\end{array}$ & Positive/negative & Positive/positive & Positive/negative & Positive/positive & Negative/positive & Poitive/negative & Negative/positive \\
\hline $\begin{array}{l}\text { EBV } \\
\text { (donor/recipient) }\end{array}$ & Positive/positive & Positive/positive & Positive/positive & Positive/positive & Positive/positive & Positive/positive & Positive/positive \\
\hline
\end{tabular}

Abbreviations: CMV, cytomegalovirus; DSA, donor-specific antibody; EBV, Epstein-Barr virus; HLA, human leukocyte antigen; NA, not available;

PRA, panel-reactive antibody.

interdisciplinary dermatopathologic conferences to rule out other differential diagnoses.

To diagnose AMR, additional evidence of elevated levels of donor-specific antibodies, along with positive $\mathrm{C} 4 \mathrm{~d}$ stain and accompanying clinical signs, was necessary. A rejection episode was defined as Banff grade II biopsy or higher, which required at least 1 of the treatment modalities described. Because we do not treat Banff grade I rejection, biopsies with Banff grades 0 and I were deemed to be no-rejection. Subclinical rejection was diagnosed on biopsy but in the absence of any clinical signs of rejection. Rejections occurring up to 2 years after transplantation were considered as early and rejections after the second postoperative year were considered to be late.

\section{Clinical Evaluation}

We analyzed every documented encounter in our hospital and outside hospitals. Clinical evaluation consisted of screening for 5 clinical signs known to indicate AR: erythema, edema, suture-line erythema, exanthema, and mucosal lesions. In addition, photographic documentation of the face allograft was performed. The presence or absence of clinical signs was primarily determined and documented by senior members of the transplantation team at the time of each patient encounter (B.P. as operating surgeon during follow-up). Subsequently, we solely included encounters during which skin tissue for biopsies of the transplanted face were obtained and peripheral venous blood samples for tacrolimus levels as an indication of immunosuppression status were collected. Ultimately, we correlated the clinical notes of each patient encounter with the photographs, tacrolimus levels, and the results of the face transplant skin biopsies.

\section{Statistical Analysis}

We generated $2 \times 2$ contingency tables according to the following criteria: (1) true-positive: biopsy showing Banff grade II or above plus presence of a clinical sign; (2) false-positive: biopsy showing Banff grade I or below plus presence of a clinical sign; (3) false-negative: biopsy showing Banff grade II or above plus absence of a clinical sign; and (4) true-negative: biopsy showing Banff grade I or below plus absence of a clinical sign.

Based on these designations, we calculated the odds ratio (OR) to determine the association of rejection with each clinical sign. The $95 \%$ CIs of the ORs were calculated according to the Baptista-Pike method. If true-positive values were 0 , the OR was deemed to be 0 . If false-positive values were 0 , the OR was deemed to be $\infty$. Statistical significance was evaluated with the Fisher exact test. Two-sided $P$ values $<.05$ were deemed to be statistically significant. The analyses were carried out for all encounters in the aggregate as well as separately with encounters stratified by time. All statistical analyses and visualization of the results were performed using GraphPad Prism, version 7.00, for MacOS (GraphPad Software).

\section{Results}

\section{Patients' Characteristics}

Five of the 7 patients were men. A detailed overview of patients' demographic details is given in the Table. The mean follow-up of this study was 66 months (range, 35-101). A total of 104 clinical encounters were identified: 46 


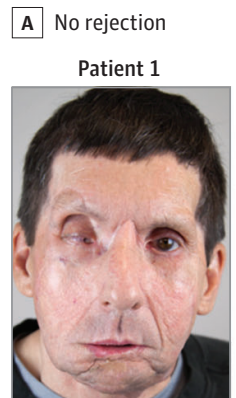

POM 42

B Early rejection

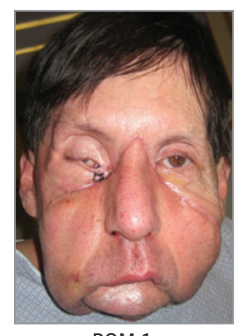

POM 1

C Late rejection

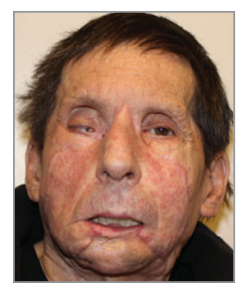

POM 83

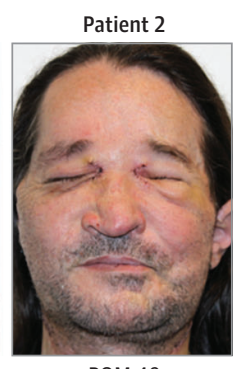

POM 40

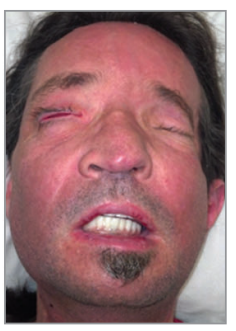

POM 22

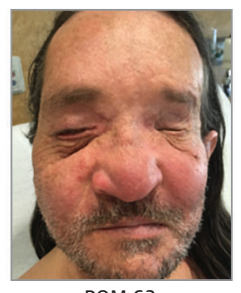

POM 63

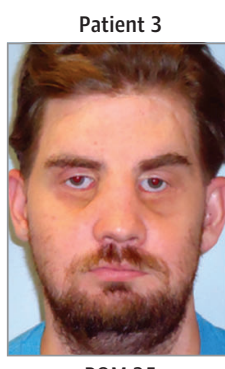

POM 25

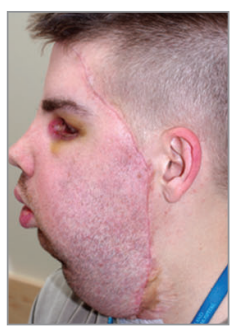

POM 1

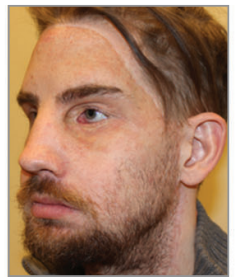

POM 34

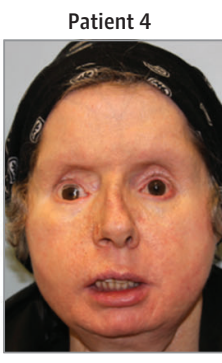

POM 42

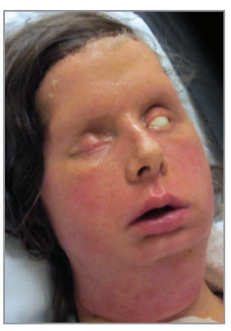

POM 2

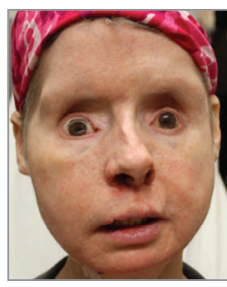

POM 59

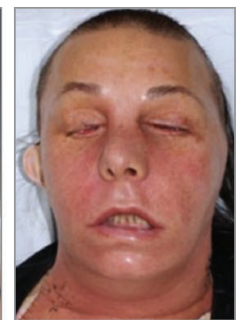

POM 1

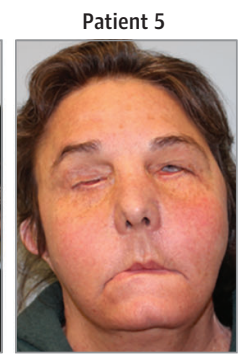

POM 26

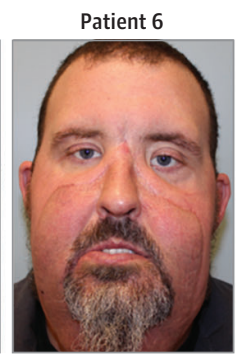

POM 21

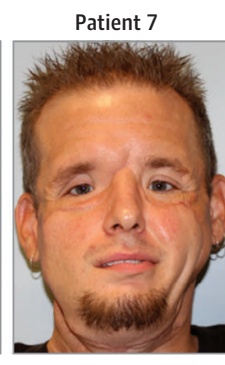

POM 9

A, No rejection. B, Early rejection ( $\leq 2$ years). C, Late rejection ( $>2$ years). At the time of writing, patient 7 had experienced no late rejection. POM indicates postoperative month.

encounters (44.2\%) represented rejection episodes and 58 encounters $(55.8 \%)$ represented no-rejection episodes. Each patient had a mean of 6.6 acute-rejection episodes (range, 2 -9). During the first 2 postoperative years, patients had a mean of 2.43 early rejections (range, $1-5$ ) and 4.14 late rejections (range, 0-7) thereafter. Of 46 AR episodes, 17 were early (37.0\%) and 29 were late (63.0\%). Of 44 TCMR episodes, 33 were graded as Banff grade III (75.0\%) and 11 were graded as Banff grade II (25.0\%). The remaining 2 rejection episodes were AMR, and both were encountered in a highly presensitized patient (patient 5) 4 years apart from each other. All rejection episodes were reversed with appropriate treatment, and no face allograft loss or patient mortality occurred. Representative photographs of patients with rejection-free FT are displayed in Figure 1A.

Clinical Signs of Acute Rejection

Typical examples of evaluated clinical signs are given in Figure 2. Erythema and edema occurred at least once in every patient during rejection. Exanthema was documented at least once in every patient except for patients 1 and 2. Mucosal lesions occurred only in patients 2,4 , and 6 . Suture-line erythema presented only in patient 1 as a sign of rejection. For the entire period, the following indicators showed a significant association with episodes of AR (Figure 3): erythema (OR, 6.33; 95\% CI, 1.48-24.15; $P$ < .001), edema (OR, 6.43; 95\% CI, 2.2316.87; $P=.001$ ), exanthema (OR, 15.0; 95\% CI, 2.3-165.7; $P=.002$ ), and subtherapeutic tacrolimus levels (OR, 2.63; 95\% CI, 1.15-5.85; $P=.03$ ). However, suture-line erythema (OR, 3.77; 95\% CI, 0.54-49.7; $P=.33$ ) and mucosal lesions (OR, 2.63; 95\% CI, 0.82-66.66; $P=.17$ ) did not show a significant association with episodes of AR.

\section{Temporal Evolution of Clinical Signs of Acute Rejection} To evaluate the reliability of clinical signs beyond 2 years posttransplantation, we performed the analysis separately for early and late rejection episodes (Figure 4). In the early period, both erythema (OR, 8.75; 95\% CI, 1.98-33.25; $P=.004$ ) and edema (OR, 21.67; 95\% CI, 3.95-87.92; $P<.001$ ) showed significant correlation with the occurrence of AR. In the late period, the 


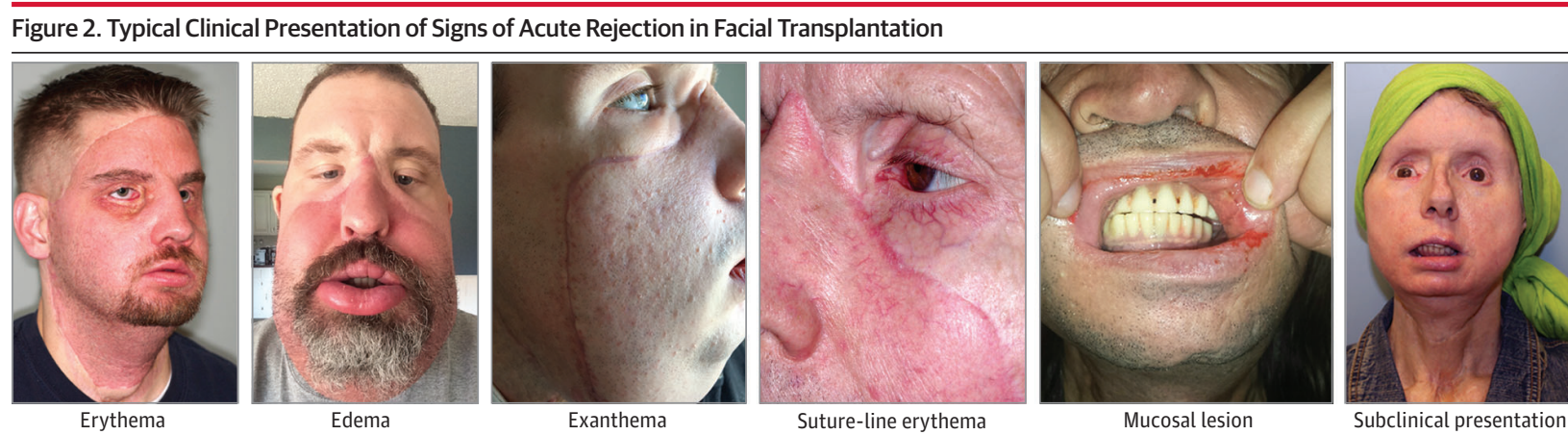

Representative clinical photographs of typical signs of acute rejection in facial transplantation: erythema, edema, exanthema, suture-line erythema, mucosa lesion, and subclinical presentation. The subclinical presentation photograph

shows the absence of any of the above-mentioned signs during a rejection episode.

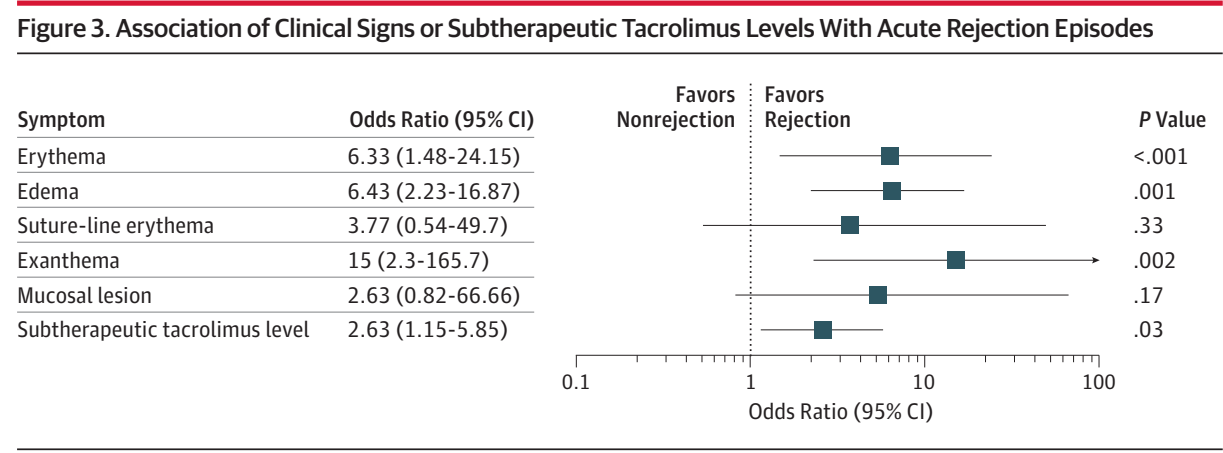

Figure 4. Association of Clinical Signs or Subtherapeutic Tacrolimus Levels With Acute Rejection Episodes Stratified by Time

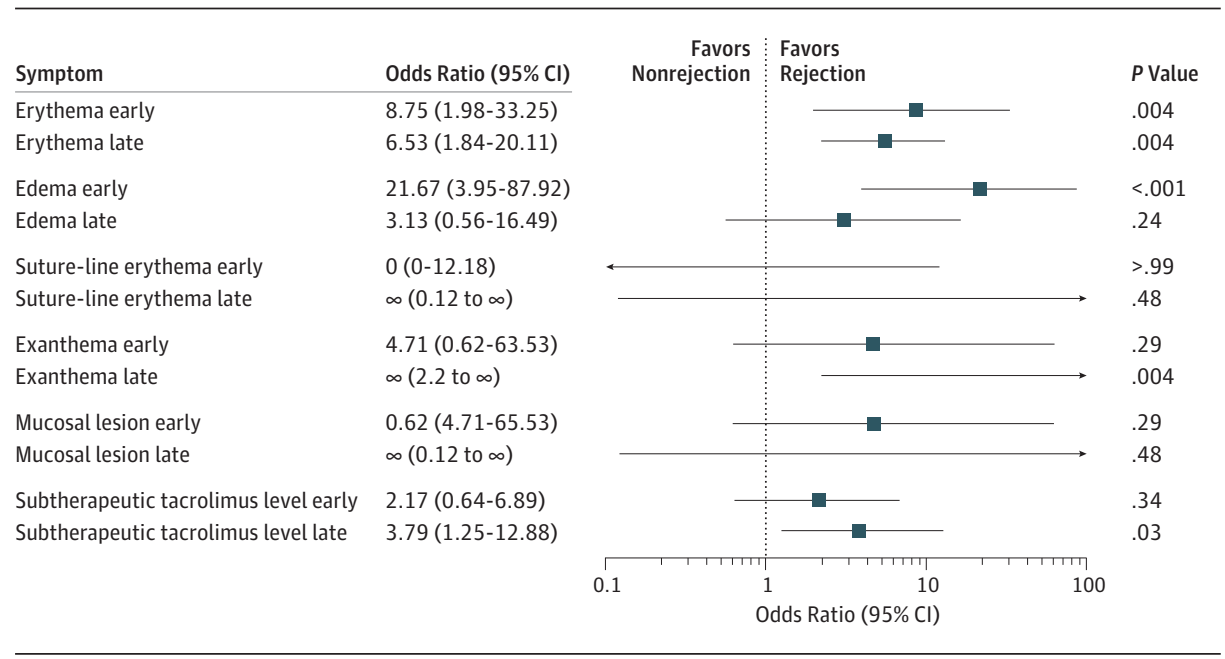

The squares represent the odds ratios and the error lines indicate the 95\% Cls.

\author{
Acute rejection episodes occurring at \\ 2 or less years and greater than 2 \\ years of follow-up were considered to \\ be early and late, respectively. The \\ squares represent the odds ratios and \\ the error lines indicate the $95 \% \mathrm{Cls}$. \\ Missing squares indicate an odds \\ ratio that equals either 0 or $\infty$.
}

presence of erythema remained statistically significant (OR, 6.53; 95\% CI, 1.84-20.11; $P=.004)$, while the presence of edema lost its statistical significance (OR, 3.13; 95\% CI, 0.56-16.49; $P=.24)$. Exanthema was not significantly correlated with AR in the early period (OR, $4.71 ; 95 \% \mathrm{CI}, 0.62-63.53 ; P=.29)$, but became statistically significant thereafter (OR, $\infty$; 95\% CI, 2.2- $\infty$; $P=.004)$. On stratification to early and late rejections, neither suture-line erythema nor mucosal lesions were significantly associated with AR. There was no statistically significant association of subtherapeutic tacrolimus levels with early rejection episodes (OR, 2.17; 95\% CI, 0.64-6.89; $P=.34$ ); however, this finding changed during late rejection episodes (OR, 3.79; 95\% CI, 1.25-12.88; $P=.03$ ). Taken together, after the first 2 years of transplantation, the only significant indicators of AR were erythema and exanthema. Furthermore, rejection was more frequently associated with subtherapeutic tacrolimus levels (Figure 4). Examples of early and late AR episodes are shown in Figure 1B and C. Furthermore, the evolution of clinical presentation of AR for every patient is summarized in the eFigure in the Supplement. 
Subclinical Rejection

Subclinical rejection occurred at least once in every patient, except for patient 6 . Taking all 46 rejection episodes into account, 8 presented subclinically (17.4\%). Of these, 1 occurred in the early period (12.5\%) and 7 occurred in the late period (87.5\%). Subclinical rejection represented $24.1 \%$ (7 rejections) of late rejection episodes but only $5.9 \%$ (1 rejection) of early rejection episodes. Five of 8 subclinical rejections (62.5\%) were associated with subtherapeutic tacrolimus levels.

\section{Discussion}

Acute rejection is the most frequently diagnosed complication of FT. Although biopsy remains mandatory for AR diagnosis, the externally exposed nature of FT skin components facilitates a clinically based assessment and underlines its importance for AR diagnosis. We demonstrated that the clinical signs of rejection evolve to less reliable markers of rejection beyond 2 years after FT. In addition, biopsy appears to be a more sensitive marker of AR because subclinical presentation of rejection is more frequent in late follow-up periods.

Currently, the FT literature does not provide much information about the diagnostic value of clinical signs over an extended period. We show that erythema appears to be a reliable clinical sign; however, the odds are decreased in the late period compared with early periods. Edema loses its importance in the late period, while exanthema gains in diagnostic value. These results are consistent with our observations that AR presents early with diffuse erythema and edema. Concurrently, we noted that these signs became more discrete and focal with progressing posttransplant time. According to our findings, mucosal lesions, as well as suture-line erythema, do not show statistically significant correlation with AR, which can be attributed mainly to the inconsistent occurrence of these signs among the patients. The limited diagnostic value is likely rooted in the co-occurrence of each of the evaluated clinical signs with dermal phenomena separated from alloimmune causes. More specifically, the presentation of mucosal lesions in patient 6 was accompanied by concomitant cytomegalovirus infection and oral candidiasis. Therefore, an infectious cause of the oral lesions cannot be ruled out in this case. Furthermore, erythema and exanthema are associated with multiple common clinical scenarios, including sunburn, allergic reactions, seborrheic dermatitis, or rosacea. ${ }^{11,18,19}$ Early allograft edema is also likely to occur with both AR and other conditions, such as lack of postsurgical lymphatic drainage, lymphadenopathy, or salivary gland leak. ${ }^{20}$ This confounding is further complicated by the subjective and qualitative nature of observer-dependent clinical evaluations. Taken together, because of the variable source of the clinical signs in FT, clinicians should carefully consider all potential differential diagnoses before commencing treatment of AR.

A potential explanation for the clinical temporality of AR may be associated with differences in the intensity of the early posttransplant alloimmune response compared with later time points. ${ }^{21,22}$ Traditionally, 3 pathways of allorecognition, including the direct, indirect, and semidirect pathways, are described in the transplant literature. ${ }^{23,24}$ The direct and semidirect pathways represent the interaction of recipient T cells with donor-derived, antigen-presenting cells or recipient antigen-presenting cells that acquired donor exosomes with major histocompatibility complex. Both processes are mostly active in the initial posttransplant phase when large amounts of donor leukocytes are transferred with the allograft and migrate to draining lymph nodes either as cells or as microvesicles. The robust inflammatory response shown by this pathway might explain the more clinically pronounced manifestations of early AR episodes. However, the indirect pathway, marked by the interaction of recipient $\mathrm{T}$ cells with recipient-derived antigen-presenting cells, is thought to predominate the late posttransplant periods and is associated with a more smoldering presentation. ${ }^{25,26}$

Yet another explanation for the temporality of AR in FT might be the role of donor $\mathrm{T}$ cells in rejection of facial allografts. Lian et $\mathrm{al}^{12}$ showed that, by examining a series of facial allograft biopsies, the targeted cell injury is spatially associated mainly with donor T cells. We hypothesized that this process is mediated through the presence of donor resident memory T cells, which interact with recipient leukocytes homing to the skin microenvironments. ${ }^{12}$ A follow-up work demonstrated that the donor $\mathrm{T}$ cells persisted for up to 1 year posttransplant and disappeared over time in FT allografts. ${ }^{27}$ Thus, if donor resident memory $\mathrm{T}$ cells play a role in the clinical presentation of $\mathrm{AR}$, it stands to reason that the robust AR presentation would be reduced as the donor $\mathrm{T}$ cells are depleted from the allograft over time, leading to a more subclinical presentation of AR.

In addition, one could speculate that a tolerogenic component plays a role in decreased intensity of clinical AR signs in late posttransplant time. Hautz et $\mathrm{al}^{28}$ studied longitudinal grade I biopsies in a series of recipients of upper extremity transplants and found that the proportion of cells stained for tolerogenic markers IDO (indoleamine 2,3-dioxygenase) and Foxp3 (forkhead box P3) increased over time, suggesting a more tolerogenic phenotype. However, more extensive studies are necessary to determine whether any regulatory mechanisms may lead to self-limitation of the AR process in FT that would result in less apparent presentation of clinical signs of rejection over the long term.

The significance and treatment response of subclinical rejection in FT still need to be determined. ${ }^{29}$ One could hypothesize that subclinical rejection, if left untreated, could progress to chronic rejection over time. Consequently, appropriate treatment of subclinical rejection may be necessary. However, given the limitations of the Banff-driven biopsy grading, such as sampling bias, intraobserver and interobserver variability, and lack of correlation to treatment response, increased patient morbidity due to overimmunosuppression should be avoided. ${ }^{25,29,30}$ Therefore, we follow a stepwise approach in treatment of subclinical rejection in our center. In the first step, we aim to adjust maintenance immunosuppression combined with topical therapy to achieve resolution of rejection. If unsatisfactory, we then proceed with intravenous immunosuppression, such as corticosteroid boluses.

Considering that most of the late subclinical rejections occurred in association with subtherapeutic tacrolimus levels, 
it also appears to be necessary to avoid target tacrolimus trough levels below $6 \mathrm{ng} / \mathrm{mL}$ in long-term treatment. In addition, the definitive association of subclinical rejection with chronic rejection still needs to be elucidated in vascularized composite allotransplantations because chronic rejection has not been formally defined. The most frequently discussed features of chronic rejection in vascularized composite allotransplantations are skin sclerosis, adnexal atrophy, and allograft vasculopathy. ${ }^{31}$ We also observe some of these chronic changes in recipients of FT in our center and are currently trying to understand the underlying histopathologic and molecular patterns. $^{32}$

Our findings suggest that AR in the FT context becomes less macroscopically ascertainable and accordingly more difficult to diagnose over time. Thus, the continued importance of protocol biopsies for immune monitoring is warranted until superior biomarkers are available to complement the rejection diagnosis. Win et $\mathrm{al}^{33}$ showed that gene expression profiling from allograft biopsies could help to distinguish between TCMR and AMR in FT. Furthermore, Kollar et $\mathrm{al}^{34}$ demonstrated that severe AR episodes in FT were associated with elevated levels of circulating matrix metalloproteinase 3 . On the other hand, rejection episodes that resolved solely with adjustment of maintenance immunosuppression or topical therapy showed significantly reduced matrix metalloproteinase 3 levels. ${ }^{34}$ These results suggest that noninvasive biomarkers could help to tailor immunosuppressive therapy in addition to biopsy and clinical findings.
Limitations

Although this study brings new insights into the clinical evolution of AR in FT, the findings should be interpreted in the context of its limitations. The limitations of this study include mainly retrospective design, single-center nature, and small sample size. With only about 44 published FTs worldwide, 7 patients represent a significant part of this limited pool. Owing to the retrospective design and emergent treatment of some AR episodes, approximately $17 \%$ of patient photographs from rejection events were not available for review. In those cases, the clinical signs could be derived solely from the clinical notes. Therefore, some intraperson and interperson variability in clinical as well as biopsy evaluation cannot be excluded. In the future, prospective multicenter studies should help to validate our findings.

\section{Conclusions}

Facial transplantation continues to show success in restoring form and function in patients with severe disfigurement of the face. To further improve the immunologic outcomes for patients with FT and vascularized composite allotransplantations in general, future research should also focus on evaluation of novel biomarkers for better diagnosis of subclinical and chronic rejection. Ultimately, these efforts should lead to bringing this unique procedure to an even larger number of patients.

\section{ARTICLE INFORMATION}

Accepted for Publication: January 22, 2019.

Published Online: April 18, 2019.

doi:10.1001/jamafacial.2019.0076

Author Contributions: Dr Haug, Dr Kollar, and Ms Obed contributed equally to the study. Dr Pomahac had full access to all of the data in the study and takes responsibility for the integrity of the data and the accuracy of the data analysis. Concept and design: Haug, Kollar, Kiwanuka, Turk, Wo, Riella, Pomahac.

Acquisition, analysis, or interpretation of data: Haug, Kollar, Obed, Kiwanuka, Turk, Wo,

Tasigiorgos, Kueckelhaus, Riella.

Drafting of the manuscript: Haug, Kollar, Kiwanuka, Turk, Riella.

Critical revision of the manuscript for important intellectual content: Haug, Kollar, Obed, Turk, Wo, Tasigiorgos, Kueckelhaus, Riella, Pomahac. Statistical analysis: Haug, Kollar, Kiwanuka, Turk, Wo.

Administrative, technical, or material support: Obed, Riella, Pomahac.

Supervision: Haug, Riella, Pomahac.

Conflict of Interest Disclosures: Dr. Kollar is the recipient of the Plastic Surgery Foundation Research Fellowship Grant outside the submitted work. Dr Tasigiorgos received grants from the United States Department of Defense during the conduct of the study. Dr Pomahac received partia salary support from a research contract with the United States Department of Defense (W911QY-09-C-0216). No other disclosures were reported.
Disclaimer: The opinions, interpretations, conclusions, and recommendations are those of the authors and are not necessarily endorsed by the Department of Defense.

Additional Contributions: We thank the Division of Dermatopathology under the leadership of George Murphy, MD, and Christine G. Lian, MD, for the histopathologic evaluation of the face transplant biopsies. The transplant coordinator, Kevin McComiskey, BSN, RN (Brigham and Women's Hospital), assisted with data collection. There was no financial compensation.

Additional Contributions: We thank the patients for granting permission to publish this information.

\section{REFERENCES}

1. Kollar B, Pomahac B. Facial restoration by transplantation. Surgeon. 2018;16(4):245-249. doi: 10.1016/j.surge.2018.01.003

2. Tasigiorgos S, Kollar B, Krezdorn N, Bueno EM, Tullius SG, Pomahac B. Face transplantationcurrent status and future developments. Transpl Int. 2018;31(7):677-688. doi:10.1111/tri.13130

3. Rifkin WJ, David JA, Plana NM, et al. Achievements and challenges in facial transplantation. Ann Surg. 2018;268(2):260-270. doi:10.1097/SLA.0000000000002723

4. Petruzzo P, Sardu C, Lanzetta M, Dubernard JM. Report (2017) of the International Registry on Hand and Composite Tissue Allotransplantation (IRHCTT). Curr Transplant Rep. 2017:4(4):294-303. doi:10.1007/s40472-017-0168-3
5. Cendales LC, Kanitakis J, Schneeberger S, et al. The Banff 2007 working classification of skin-containing composite tissue allograft pathology. Am J Transplant. 2008;8(7):1396-1400. doi:10.1111/j.1600-6143.2008.02243.x

6. Fischer S, Lian CG, Kueckelhaus M, et al. Acute rejection in vascularized composite allotransplantation. Curr Opin Organ Transplant. 2014;19(6):531-544. doi:10.1097/MOT 0000000000000140

7. Petruzzo P, Lanzetta M, Dubernard J-M, et al. The International Registry on Hand and Composite Tissue Transplantation. Transplantation. 2010;90 (12):1590-1594. doi:10.1097/TP.0b013e3181ff1472

8. Bergfeld W, Klimczak A, Stratton JS, Siemionow MZ. A four-year pathology review of the near total face transplant. Am J Transplant. 2013;13(10):27502764. doi:10.1111/ajt.12379

9. Chaudhry A, Sosin M, Bojovic B, Christy MR, Drachenberg CB, Rodriguez ED. Defining the role of skin and mucosal biopsy in facial allotransplantation: a 2-year review and analysis of histology. Plast Reconstr Surg. 2015;136(3):559-567. doi:10. 1097/PRS.0000000000001529

10. Kaufman CL, Marvin MR, Chilton PM, et al. Immunobiology in VCA. Transpl Int. 2016;29(6): 644-654. doi:10.1111/tri.12764

11. Kanitakis J. The challenge of dermatopathological diagnosis of composite tissue allograft rejection: a review. J Cutan Pathol. 2008;35(8): 738-744. doi:10.1111/j.1600-0560.2007.00889.x 12. Lian CG, Bueno EM, Granter SR, et al. Biomarker evaluation of face transplant rejection: association 
of donor T cells with target cell injury. Mod Pathol. 2014;27(6):788-799. doi:10.1038/modpathol.2013. 249

13. Pomahac B, Pribaz J, Eriksson E, et al. Three patients with full facial transplantation. N Engl J Med. 2012;366(8):715-722. doi:10.1056/NEJMoa1111432

14. Borges TJ, O'Malley JT, Wo L, et al. Codominant role of interferon- $\gamma$ - and interleukin-17-producing $T$ cells during rejection in full facial transplant recipients. Am J Transplant. 2016;16(7):2158-2171. doi:10.1111/ajt.13705

15. Diaz-Siso JR, Fischer S, Sisk GC, et al. Initial experience of dual maintenance immunosuppression with steroid withdrawal in vascular composite tissue allotransplantation. Am J Transplant. 2015;15(5):1421-1431. doi:10.1111/ajt.13103

16. Krezdorn N, Murakami N, Pomahac B, Riella LV. Immunological characteristics of a patient with belatacept-resistant acute rejection after face transplantation. Am J Transplant. 2016;16(11):3305 3307. doi:10.1111/ajt.13977

17. Chandraker A, Arscott R, Murphy GF, et al. The management of antibody-mediated rejection in the first presensitized recipient of a full-face allotransplant. Am J Transplant. 2014;14(6):14461452. doi:10.1111/ajt.12715

18. Pomahac B, Pribaz J, Eriksson E, et al. Restoration of facial form and function after severe disfigurement from burn injury by a composite facial allograft. Am J Transplant. 2011;11(2):386-393. doi:10.1111/j.1600-6143.2010.03368.x

19. Kanitakis J. Transmission of rosacea from the graft in facial allotransplantation. Am J Transplant 2011;11(6):1338-1339. doi:10.1111/j.1600-6143.2011. 03507.x
20. Lee TC, Chansakul T, Huang RY, et al. Early postoperative imaging and image-guided procedures on patients with face transplants. AJNR Am J Neuroradiol. 2015;36(3):568-574. doi:10.3174/ ajnr.A4141

21. Sicard A, Kanitakis J, Dubois V, et al. An integrated view of immune monitoring in vascularized composite allotransplantation. Curr Opin Organ Transplant. 2016;21(5):516-522. doi:10 1097/МOT.0000000000000354

22. Siemionow M, Nasir S. Immunologic responses in vascularized and nonvascularized skin allografts. J Reconstr Microsurg. 2008;24(7):497-505. doi 10.1055/s-0028-1088232

23. Afzali B, Lombardi G, Lechler RI. Pathways of major histocompatibility complex allorecognition. Curr Opin Organ Transplant. 2008;13(4):438-444 doi:10.1097/MOT.0b013e328309ee31

24. Marino J, Paster J, Benichou G. Allorecognition by $T$ lymphocytes and allograft rejection. Front Immunol. 2016;7:582. doi:10.3389/fimmu.2016. 00582

25. Sarhane KA, Tuffaha SH, Broyles JM, et al. A critical analysis of rejection in vascularized composite allotransplantation: clinical, cellular and molecular aspects, current challenges, and novel concepts. Front Immunol. 2013;4:406. doi:10. 3389/fimmu.2013.00406

26. Sarhane KA, Khalifian S, Ibrahim Z, et al. Diagnosing skin rejection in vascularized composite allotransplantation: advances and challenges. Clin Transplant. 2014;28(3):277-285. doi:10.1111/ctr.12316

27. Win T, Murakami N, Avraham-Davidi I, et al. Contribution of donor versus recipient $T$ cells in face transplant rejection [abstract]. Am J Transplant. 2017;17(suppl 3):611. https://atcmeetingabstracts com/abstract/contribution-of-donor-versusrecipient-t-cells-in-face-transplant-rejection/

28. Hautz $T$, Zelger $B$, Brandacher $G$, et al. Histopathologic characterization of mild rejection (grade I) in skin biopsies of human hand allografts. Transpl Int. 2012;25(1):56-63. doi:10.1111/j.14322277.2011.01369.x

29. Schneider M, Cardones ARG, Selim MA, Cendales LC. Vascularized composite allotransplantation: a closer look at the Banff working classification. Transpl Int. 2016;29(6):663671. doi:10.1111/tri.12750

30. Starzl R, Brandacher G, Lee WPA, et al. Review of the early diagnoses and assessment of rejection in vascularized composite allotransplantation. Clin Dev Immunol. 2013;2013:402980. doi:10.1155/ 2013/402980

31. Morelon E, Petruzzo P, Kanitakis J. Chronic rejection in vascularized composite allotransplantation. Curr Opin Organ Transplant. 2018;23(5):582-591. doi:10.1097/MOT. 0000000000000571

32. Krezdorn N, Lian CG, Wells M, et al. Chronic rejection of human face allografts. Am J Transplant. 2018;(October). doi:10.1111/ajt.15143

33. Win TS, Murakami N, Borges TJ, et al. Longitudinal immunological characterization of the first presensitized recipient of a face transplant. $J \mathrm{Cl}$ Insight. 2017;2(13):e93894. doi:10.1172/jci.insight. 93894

34. Kollar B, Shubin A, Borges TJ, et al. Increased levels of circulating MMP3 correlate with severe rejection in face transplantation. Sci Rep. 2018;8(1): 14915. doi:10.1038/s41598-018-33272-7 